Author affiliations and support information (if applicable) appear at the end of this article.

Published at jco.org on April 12, 2017.

Clinical trial information:

ISRCTN73030316.

Corresponding author: Thomas Powles, MRCP, MD, Experimental Cancer Medicine Centre, Barts Cancer Institute, Barts Health NHS Trust and the Royal Free NHS Trust, Queen Mary University of London, London EC1A7BE, United Kingdom; e-mail: thomas.powles@ bartshealth.nhs.uk

(C) 2017 by American Society of Clinical Oncology

0732-183X/17/3516w-1770w/\$20.00

\title{
Randomized Phase II Study Investigating Pazopanib Versus Weekly Paclitaxel in Relapsed or Progressive Urothelial Cancer
}

Robert J. Jones, Syed A. Hussain, Andrew S. Protheroe, Alison Birtle, Prabir Chakraborti, Robert A. Huddart, Satinder Jagdev, Amit Bahl, Andrew Stockdale, Santhanam Sundar, Simon J. Crabb, Judith Dixon-Hughes, Laura Alexander, Anna Morris, Caroline Kelly, Jon Stobo, James Paul, and Thomas Powles

\section{$\begin{array}{llllllll}\text { A } & \text { B } & \text { S } & \text { T } & \text { R } & \text { A } & \text { C } & \text { T }\end{array}$}

\section{Purpose}

Two previous single-arm trials have drawn conflicting conclusions regarding the activity of pazopanib in urothelial cancers after failure of platinum-based chemotherapy.

\section{Patients and Methods}

This randomized (1:1) open-label phase II trial compared the efficacy of pazopanib $800 \mathrm{mg}$ orally with paclitaxel ( $80 \mathrm{mg} / \mathrm{m}^{2}$ days 1,8 , and 15 every 28 days) in the second-line setting. The primary end point was overall survival (OS).

\section{Results}

Between August 2012 and October 2014, 131 patients, out of 140 planned, were randomly assigned. The study was terminated early on the recommendation of the independent data monitoring committee because of futility. Final analysis after the preplanned number of deaths ( $n=110$ ) occurred after a median follow-up of 18 months. One hundred fifteen deaths had occurred at the final data extract presented here. Median OS was 8.0 months for paclitaxel $180 \%$ $\mathrm{Cl}, 6.9$ to 9.7 months) and 4.7 months for pazopanib ( $80 \% \mathrm{Cl}, 4.2$ to 6.4 months). The hazard ratio $(\mathrm{HR})$ adjusted for baseline stratification factors was $1.28(80 \% \mathrm{Cl}, 0.99$ to 1.67; one-sided $P=.89)$. Median progression-free survival was 4.1 months for paclitaxel $(80 \% \mathrm{Cl}, 3.0$ to 5.6 months) and 3.1 months for pazopanib $(80 \% \mathrm{Cl}, 2.7$ to 4.6 months; $\mathrm{HR}, 1.09 ; 80 \% \mathrm{Cl}, 0.85$ to 1.40 ; onesided $P=.67)$. Discontinuations for toxicity occurred in $7.8 \%$ and $23.1 \%$ for paclitaxel and pazopanib, respectively.

\section{Conclusion}

Pazopanib did not have greater efficacy than paclitaxel in the second-line treatment of urothelial cancers. There was a trend toward superior OS for paclitaxel.

\section{J Clin Oncol 35:1770-1777. (c) 2017 by American Society of Clinical Oncology}

\section{INTRODUCTION}

Patients with advanced/metastatic urothelial cancer (UC) are initially treated with platinum-based chemotherapy. ${ }^{1,2}$ This results in responses and subsequent clinical benefit. However, the vast majority of these patients relapse and die as a result of their disease. When relapse occurs after initial chemotherapy, further chemotherapy, such as paclitaxel, docetaxel, or vinflunine, is recommended. ${ }^{3,4}$ Vinflunine is licensed in Europe in this setting. However, other agents are more widely used globally. ${ }^{3,5,6}$ At the time this study started, no chemotherapy agents were approved in this setting in the United States.
Outcomes for these patients are particularly poor, with a median survival in the region of 8 months. ${ }^{3}$ To date, no regimen has a proven survival advantage over best supportive care in randomized trials. ${ }^{6}$ Results from randomized trials with immune therapies in this setting are awaited.

There is in vitro and clinical rationale for targeting vascular endothelial growth factor (VEGF) in UC. ${ }^{7,8}$ Pazopanib is a VEGF receptor tyrosine kinase inhibitor with a favorable toxicity profile. ${ }^{9}$ It is licensed in a number of malignancies, including renal cancer. ${ }^{9}$ Preliminary data from two single-arm phase II studies in platinum-refractory metastatic UC are contradictory. ${ }^{10,11}$ Clinical benefit was as high as $80 \%$ in one study, whereas the other showed 2016.70.7828 
limited activity with no responses. To clarify this issue and plan for a randomized phase III study, the United Kingdom Bladder Cancer Clinical Studies Group tested pazopanib against weekly paclitaxel in a randomized phase II study. The control arm was chosen on the basis of previously published phase II data and a survey, before the study, which showed that paclitaxel was the most widely used drug in this setting in the $\mathrm{UK}^{3,12}$

\section{PATIENTS AND METHODS}

\section{Patients}

Patients participating in this study were required to have: histologically confirmed transitional-cell carcinoma of the bladder, renal pelvis, ureter, or urethra that was locally advanced or metastatic (T4b and/or N1-3 and/or M1); progressive disease during or after one prior platinum-based chemotherapy regimen for advanced disease (patients may have had two platinum-containing regimens if one of these was administered as adjuvant/neoadjuvant treatment), with progression of disease determined radiologically by sites; measurable disease by Response Evaluation Criteria in Solid Tumors (RECIST) 1.1; adequate organ function (bone marrow, liver, and renal function); signed and dated informed consent; and an Eastern Cooperative Oncology Group performance status of 0 or 1 . Patients with established cardiac or GI disease, uncontrolled hypertension, a recent history of bleeding, thrombotic events, or major surgery (within 6 weeks) were excluded from the study. Patients with untreated brain metastasis, significant prior malignancy, or prior exposure to taxane chemotherapy or VEGF-targeted therapy were also excluded.

\section{Outcome Measures}

Overall survival (OS) from date of randomization was the primary outcome measure. Secondary outcome measures included toxicity according to Common Terminology Criteria for Adverse Events v4.02, response rate (RR) by RECIST 1.1, progression-free survival (PFS), and clinical benefit rate (CBR; proportion of patients with complete response, partial response, or stable disease) 12 and 24 weeks after start of treatment.

Quality of life was assessed using the validated bladder cancerspecific tool, Functional Assessment of Cancer Therapy-Bladder (FACT$\mathrm{Bl}$ ), which assesses physical, social/family, emotional, and functional well-being and additional concerns domains to give an overall score. It also combines the physical, functional, and cancer-specific subscales (those most likely to change in a chemotherapy clinical trial) to give a trial outcome index.

\section{Randomization, Treatment, and Follow-Up}

This was a two-arm, open-label randomized (1:1) phase II study (see supplementary data). A computerized algorithm, which used a minimization approach ${ }^{13}$ and incorporated a random component to avoid predictability, was used to randomly assign patients and ensure that the groups were well matched with respect to the following factors: response to previous treatment (time to progression: $\leq 6$ months $v>6$ months), presence of visceral and/or bone metastasis, performance status, and investigational site. Patients in the control arm received paclitaxel $80 \mathrm{mg} / \mathrm{m}^{2}$ by intravenous infusion on days 1,8 , and 15 of a 28 -day cycle, with a maximum duration of 24 weeks. Dose reductions (to 70 then $60 \mathrm{mg} / \mathrm{m}^{2}$ ) and interruptions were permitted to manage toxicity. Patients in the investigational arm received pazopanib $800 \mathrm{mg}$ orally once daily until progression. Dose reductions according to the manufacturer's instruction and interruptions (up to 14 days) were permitted to manage toxicity. Patients were assessed every four weeks for the first 24 weeks and then sixweekly. Cross-section imaging to assess RR and PFS were performed 12-weekly until progression.

\section{Statistical Consideration and Oversight}

The study was designed to detect a $50 \%$ improvement in median overall survival among patients receiving pazopanib compared with paclitaxel (improvement from 8 months to 12 months with $90 \%$ power, $20 \%$ one-sided level of statistical significance, or equivalently with $80 \%$ power at the $10 \%$ level of statistical significance). This required 110 events, which could be achieved with 140 patients ( 70 per arm). The study design was based around a phase II screening design, with the three-outcome design forming the basis of the decisionmaking process. ${ }^{14,15}$ A result favoring pazopanib that was significant at the one-sided $10 \%$ level would suggest that a subsequent phase III trial should be performed. A result in favor of pazopanib that was statistically significant at the one-sided $20 \%$ level, but not at the onesided $10 \%$ level, would require other supportive data in terms of a statistically significant improvement in PFS, at the $10 \%$ level, to indicate a phase III study was warranted. A result not reaching statistical significance at the $20 \%$ level would suggest that no further investigation of pazopanib should be performed in this setting.

The primary analysis of the overall survival end point was conducted on an intention-to- treat basis. OS and PFS were compared between the study arms in the context of the hazard ratio (HR) from a Cox model incorporating study arm and the factors used in the minimization algorithm. A test for interaction was conducted to assess whether any observed effect of the study intervention depended on the other clinical factors used in the minimization algorithm. The final analysis was planned at the end of the minimum follow-up period once the required number of events (110 deaths) had been observed. A Kaplan-Meier curve was used to illustrate the relative OS in the two treatment arms. Toxicity was compared between the study arms using a Mann-Whitney test. Clinical benefit rate was compared using the odds ratio (OR) from a logistic regression model incorporating study arm and the factors used in the minimization algorithm. Quality-of-life data were calculated and interpreted using FACT-Bl scoring and interpretation materials (http://www.facit.org/FACITOrg/Questionnaires) and assessed using area under the curve (AUC) techniques. ${ }^{16}$ Missing values were filled in using interpolation or last value carried forward, as appropriate. Missing baseline values were imputed with the earliest available value. The calculated AUC scores were standardized by the quality-of-life assessment duration, which was time to progression, death, or 97 weeks, whichever was shortest. These standardized AUC scores were adjusted by subtracting the cycle one/week one value. To adjust for multiple testing, the false discovery rate (FDR) was calculated using the p.adjust function (fdr option) of the stats library in R. ${ }^{17}$

The trial was open labeled, and an independent data monitoring committee monitored the adverse events (for toxicity) and efficacy (for futility). A trial steering committee convened on a regular basis. The trial had appropriate ethical and regulatory approval (ISRCTN73030316).

\section{RESULTS}

Between August 2012 and October 2014, 131 patients were randomly assigned from 24 United Kingdom sites, 65 to receive paclitaxel and 66 to receive pazopanib. Recruitment was terminated prematurely (October 14, 2014) on recommendation of the independent data monitoring committee after an interim analysis of the first 60 deaths in the study showed that the trial was unlikely to achieve the objective of demonstrating superiority for pazopanib compared with paclitaxel. At this time, 131 of 140 planned patients had been enrolled.

Investigators were informed of this decision immediately, but patients were permitted to continue with their allocated study treatment if both the patient and the investigator believed it was in the patient's best interests. Patient distribution in the trial is 


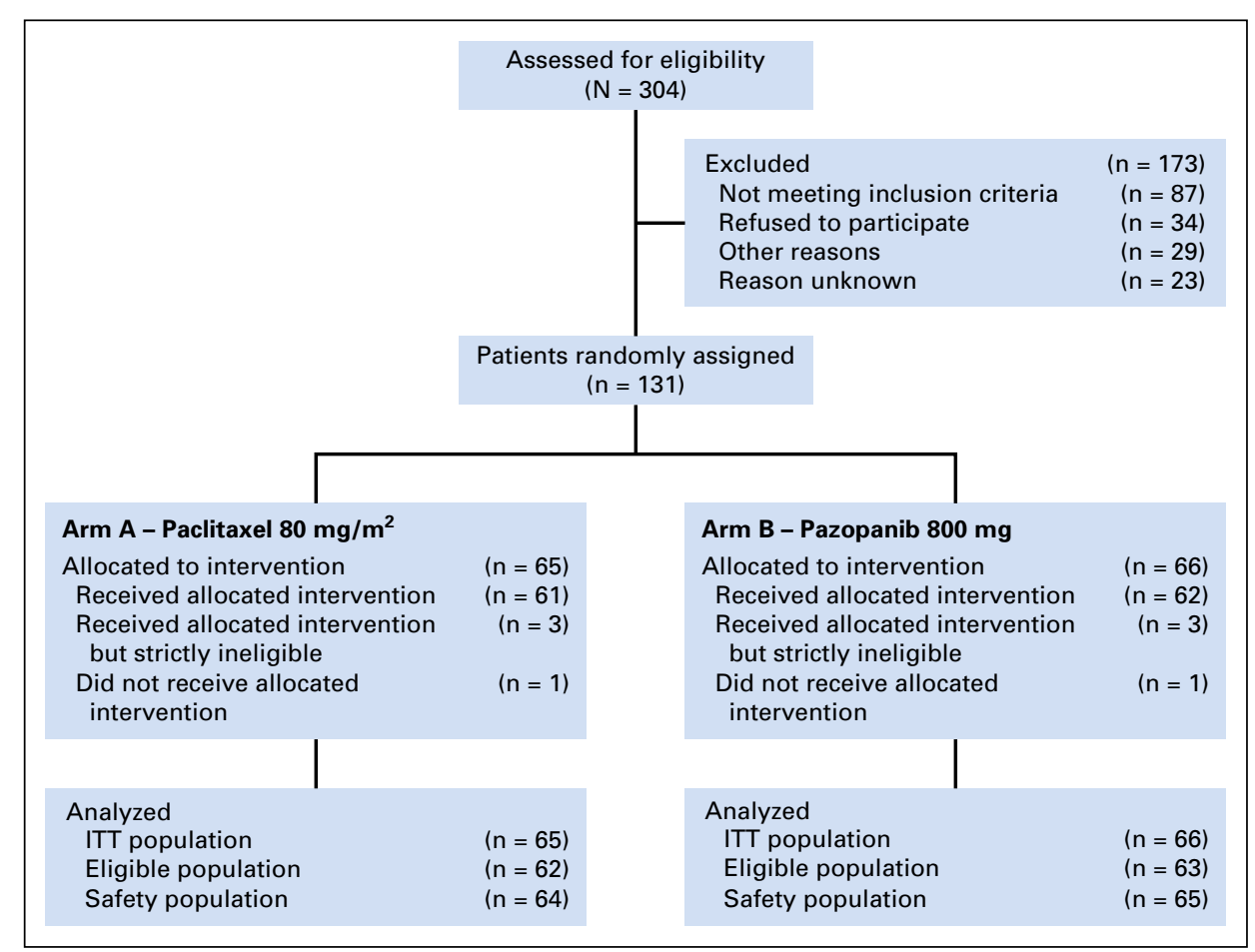

Fig 1. CONSORT diagram. ITT, intent to treat.

illustrated in Figure 1 (CONSORT diagram). No patients stopped pazopanib because of futility of the trial. At the time of final data extraction (May 20, 2016), there were 115 OS events. The median follow-up of the patients was 18 months.

\section{Patient Characteristics}

Patient characteristics are listed in Table 1 . Notably, only two patients had prior cystectomy; $24.4 \%$ had liver metastases, and $61 \%$ had impaired performance status.

\section{Efficacy}

Median OS from randomization was 8.0 months $(80 \% \mathrm{CI}$, 6.9 to 9.7 months) and 4.7 months ( $80 \%$ CI, 4.2 to 6.4 months) for paclitaxel and pazopanib, respectively (adjusted HR, 1.28; $80 \% \mathrm{CI}, 0.99$ to 1.67 ; one-sided $P=.89$; two-sided $P=.23$ ). Median PFS was 4.1 months ( $80 \%$ CI, 3.0 to 5.6 months) and 3.1 months ( $80 \% \mathrm{CI}, 2.7$ to 4.6 months) for paclitaxel and pazopanib, respectively (adjusted HR, 1.09; 80\% CI, 0.85 to 1.40; one-sided $P=.67$; two-sided $P=.66$ ). Kaplan-Meier survival distributions are show in Figure 2 (Kaplan-Meier

\begin{tabular}{|c|c|c|}
\hline Characteristic & Paclitaxel $(n=65)$ & Pazopanib $(n=66)$ \\
\hline Male & 75 & 67 \\
\hline Age, years & $70(63-77)$ & $69(61-75)$ \\
\hline \multicolumn{3}{|l|}{ Ethnicity } \\
\hline White & 91 & 95 \\
\hline Asian & 3 & 2 \\
\hline Afro-Caribbean & 2 & 2 \\
\hline Other & 4 & 1 \\
\hline \multicolumn{3}{|l|}{ ECOG performance status } \\
\hline 0 & 39 & 39 \\
\hline 1 & 52 & 53 \\
\hline 2 & 9 & 8 \\
\hline Bladder primary & 66 & 71 \\
\hline T4 disease at diagnosis & 20 & 15 \\
\hline Liver metastases & 29 & 20 \\
\hline Nodal metastases & 45 & 53 \\
\hline Time since last platinum therapy to randomization, days & $146(65-244)$ & $161(81-273)$ \\
\hline$\leq 6$ months from previous treatment to progression & 75 & 73 \\
\hline Time from initial diagnosis to randomization, days & $444(325-661)$ & $478(300-781)$ \\
\hline
\end{tabular}


A

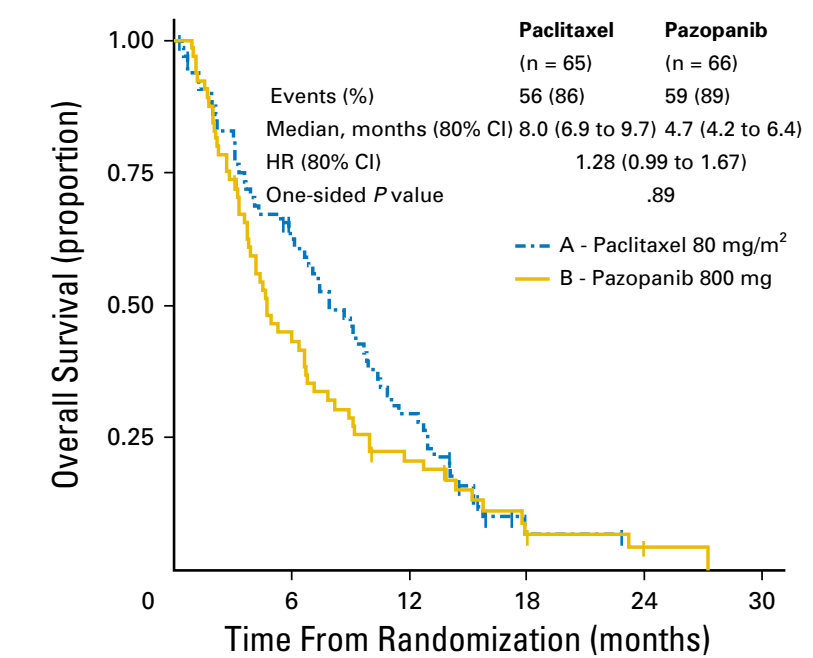

No. at risk:

A - Paclitaxel
$80 \mathrm{mg} / \mathrm{m}^{2}$

B - Pazopanib $800 \mathrm{mg}$
B

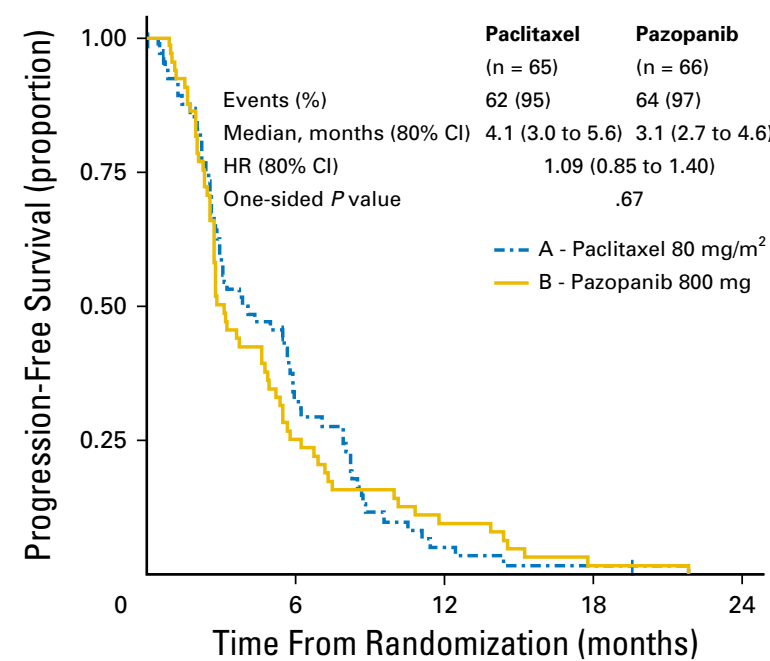

No. at risk:

$\begin{array}{rccccccccc}\text { A - Paclitaxel } & 65 & 38 & 20 & 7 & 3 & 1 & 1 & 0 & 0 \\ \begin{array}{r}80 \mathrm{mg} / \mathrm{m}^{2} \\ \text { B - Pazopanib }\end{array} & 66 & 32 & 16 & 10 & 6 & 3 & 1 & 1 & 0 \\ 800 \mathrm{mg} & & & & & & & & \end{array}$

Fig 2. Kaplan-Meier survival distribution for (A) overall survival, and (B) progression-free survival used on the intent-to-treat (ITT) population using the Kaplan-Meier methodology. Hazard ratios (HRs) were calculated using the Cox model and factors in the minimization algorithm.

curves for OS and PFS). OS and PFS by preplanned subgroups are illustrated in Figure 3. Nine (13.9\%) of 65 and three $(4.5 \%)$ of 66 patients had complete or partial response at 12 weeks in the paclitaxel and pazopanib arms, respectively. The CBR (proportion of patients with complete response, partial response, or stable disease) at 12 weeks was $40 \%$ for the paclitaxel arm and $36.4 \%$ for pazopanib (adjusted OR, $1.27 ; 80 \%$ CI, 0.78 to 2.06 ; one-sided $P=.73$; two-sided $P=.54)$. At 24 weeks, the CBR was $18.5 \%$ and $12.1 \%$ respectively (adjusted OR, 1.76 ; $80 \% \mathrm{CI}, 0.92$ to 3.39 ; one-sided $P=.87$; two-sided $P=.27)$.

\section{Treatment Exposure and Discontinuation}

Overall, 22 patients $(34.4 \%)$ in the control arm received the planned six cycles of paclitaxel; the median number of cycles was four. The median time on pazopanib was 10.9 weeks ( $80 \% \mathrm{CI}, 9.9$ to 11.9 weeks). Five patients $(7.8 \%)$ and 15 patients $(23.1 \%)$ discontinued treatment because of toxicity, and 28 patients $(43.8 \%)$ and 40 patients $(61.5 \%)$ discontinued because of disease progression for paclitaxel and pazopanib, respectively. Sixteen patients $(25 \%)$ and 21 patients $(32.3 \%)$ required one or more dose reductions for paclitaxel and pazopanib, respectively.

\section{Treatment-Emergent Adverse Events}

Thirty-nine percent and $51 \%$ of patients experienced one or more high-grade ( 3 or 4 ) treatment-emergent adverse events (AEs) during the study treatment period; $27 \%$ and $38 \%$ experienced one or more high-grade AEs that were attributed to the study drug (for paclitaxel and pazopanib, respectively). The most common treatment-emergent AEs are summarized in
Table 2. Toxicity was in line with expected AE profiles, with neuropathy and neutropenia more prominent with paclitaxel and hypertension, diarrhea, and transaminitis more prominent with pazopanib. The most common AEs resulting in discontinuation of pazopanib were fatigue, nausea, and vomiting.

\section{Quality of Life}

Seventeen patients were excluded from the FACT-Bl AUC analyses, because of a lack of data (usually incomplete or absence of completed questionnaires [76\%]). FACT-Bl trial outcome index is significantly reduced in the pazopanib arm (baseline adjusted standardized AUC median, -2.7; interquartile range (IQR), -10.3 to 0.0 ) compared with paclitaxel (baseline adjusted standardized AUC median, 0.0; IQR, -4.9 to 2.0); two-sided $P=.0028$ (FDR adjusted $P=.0034$ ). Similarly, FACT-Bl total score is also significantly reduced with pazopanib (baseline adjusted standardized AUC median, -3.8; IQR, -9.8 to 0.0 ) compared with paclitaxel (baseline adjusted standardized AUC median, 0.0; IQR, -5.2 to 0.8 ); two-sided $P=.0034$ (FDR adjusted $P=.0034$ ). The change from baseline in each quality of life measure is illustrated in Figure 4.

\section{Subsequent Therapies}

Twenty percent and $24 \%$ of patients received further chemotherapy, $15 \%$ and $18 \%$ received palliative radiotherapy, and $6 \%$ and $0 \%$ received an immune checkpoint inhibitor after completing study treatment in the paclitaxel and pazopanib arms, respectively. Of the four patients receiving immune checkpoint inhibitors, one received nivolumab and three received atezolizumab. 


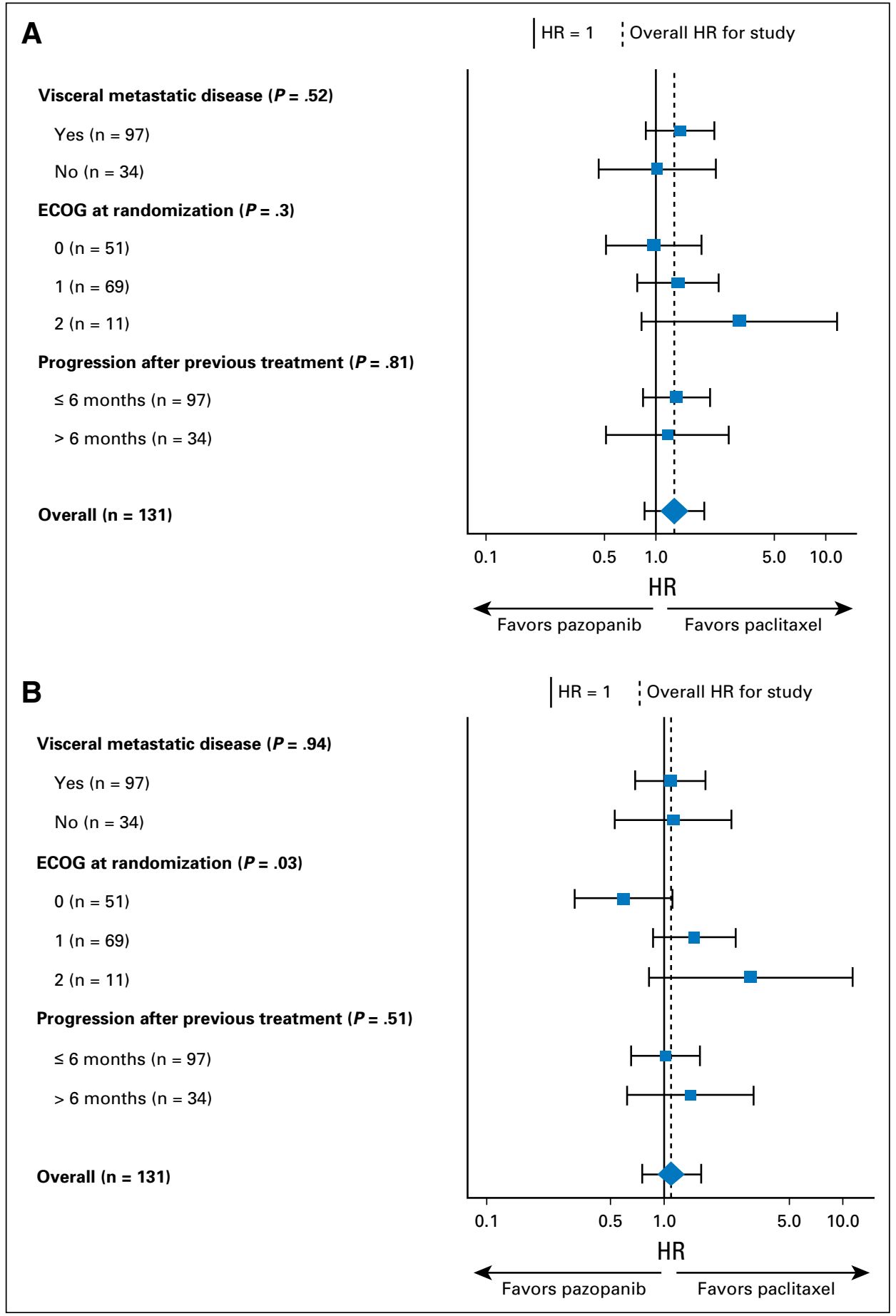

Fig 3. Forest plot of (A) overall survival, and (B) progression-free survival by subgroup. Hazard ratios (HRs) were calculated using Cox model incorporating study arm and the factors used in the minimization algorithm. ECOG, Eastern Cooperative Oncology Group.

\section{DISCUSSION}

There is no globally accepted standard of care in second-line treatment of advanced UC after the failure of prior platinumbased chemotherapy. ${ }^{1}$ A variety of chemotherapy drugs have demonstrated modest activity in small proportions of patients, but none have demonstrated conclusive clinical benefits compared with best supportive care. ${ }^{3}$ It is possible that such benefits exist among a defined subgroup of patients, but there are no data to guide us in making such a selection. Vinflunine is licensed in Europe despite no survival benefit over best supportive care in the intention-to-treat population. ${ }^{6}$ The OS of these patients was 6.9 months, with a nonsignificant $22 \%$ reduction in the risk of death over best supportive care. Other agents, such as single-agent taxanes or chemotherapy doublets, have been investigated in single-arm trials without success. Meta-analysis suggests doublets are not superior to single-agent therapy. ${ }^{18}$ Median OS for patients in these 


\begin{tabular}{|c|c|c|c|c|c|}
\hline \multirow[b]{2}{*}{ Toxicity } & \multicolumn{2}{|c|}{ Paclitaxel $(n=64)$} & \multicolumn{2}{|c|}{ Pazopanib $(n=65)$} & \multirow[b]{2}{*}{$P^{*}$} \\
\hline & Any Grade ( $\geq 1$ ) & Grade $\geq 3$ & Any Grade ( $\geq 1$ ) & Grade $\geq 3$ & \\
\hline Any adverse event & 94 & 27 & 91 & 39 & - \\
\hline Neutropenia & 36 & 6 & 9 & 0 & .001 \\
\hline Thrombocytopenia & 2 & 0 & 9 & 2 & .32 \\
\hline Hypophosphatemia & 38 & 6 & 18 & 0 & .010 \\
\hline Elevated alanine transaminase & 34 & 2 & 48 & 9 & .056 \\
\hline Hypertension & 0 & 0 & 20 & 9 & $<.001$ \\
\hline Alopecia & 41 & 0 & 2 & 0 & $<.001$ \\
\hline Nausea & 41 & 0 & 42 & 3 & .49 \\
\hline Anorexia & 16 & 0 & 31 & 0 & .026 \\
\hline Diarrhea & 27 & 2 & 49 & 6 & .007 \\
\hline Altered taste & 5 & 0 & 14 & 0 & .003 \\
\hline Fatigue & 72 & 5 & 74 & 9 & .56 \\
\hline Mucositis & 20 & 2 & 15 & 0 & .50 \\
\hline Neuropathy & 38 & 2 & 3 & 0 & $<.001$ \\
\hline PPE & 0 & 0 & 11 & 2 & .13 \\
\hline
\end{tabular}

chemotherapy trials remains well below 10 months and is usually closer to 7 months. ${ }^{3}$

Recent early-phase single-arm trials of immune checkpoint inhibitors have clearly demonstrated efficacy for these drugs in a significant proportion of patients. ${ }^{19}$ Atezolizumab is an inhibitor of programmed death-ligand 1. Results from a large phase II study in a chemotherapy-refractory UC population showed significant activity, particularly in tumors that overexpress the programmed death-ligand 1 biomarker in the immune component of the tumor (RR, 27\%; 95\% CI, $19 \%$ to $37 \%$ ). ${ }^{20}$ Overall survival for the entire cohort was 7.9 months $(6.7, \mathrm{NE})$. Atezolizumab is licensed in both biomarker-positive and -negative patients in the United States in this setting. A recent randomized phase III trial showed that pembrolizumab, an inhibitor of programmed death-1, prolonged survival compared with chemotherapy agents, including taxanes, in platinum-refractory $\mathrm{UC}(\mathrm{HR}, 0.73 ; 95 \% \mathrm{CI}, 0.59$ to 0.91 ; $P=.002)^{21}$

The results presented here show pazopanib is not better than weekly paclitaxel. Indeed, the trial was stopped with nine patients still to recruit after a futility analysis indicated that the trial would not meet its primary objective of demonstrating superiority of pazopanib over paclitaxel. Final results showed a trend toward an OS benefit for paclitaxel, with an HR of $1.28(80 \% \mathrm{CI}, 0.99$ to 1.67; one-sided $P=.89$; two-sided $P=.23$ ) in favor of paclitaxel. Other efficacy results for paclitaxel were in line with previous results for chemotherapy in the second-line setting (RR, 14\%; and median PFS, 4.1 months), and the fractionated chemotherapy schedule used here was generally well tolerated. The reasons for the relative lack of efficacy for pazopanib remain unclear. Previous data with pazopanib in this setting were contradictory, highlighting shortcomings of single-arm single-institution trials in UC, where patient baseline characteristics have a significant effect on outcome. The data presented here support the study by Pili et $\mathrm{al}^{11}$ suggesting limited activity. Pazopanib was administered at standard doses and was relatively well tolerated. However, $23 \%$ of patients stopped therapy because of toxicity, compared with $8 \%$ of those on chemotherapy. Thus, lack of exposure to study drug may have partly contributed to the relatively poor outcomes for those receiving pazopanib. Pharmacokinetic studies were not performed in this study, but there is no reason to suggest the lack of efficacy was dose related ( $32 \%$ of patients required a dose reduction, which is in line with those seen with pazopanib in other settings).

VEGF-targeted therapy has been investigated previously in UC with mixed results. Single-arm trials are difficult to interpret, as described above. Three randomized trials are noteworthy. The first is a phase II trial with ramucirumab (an anti-VEGF receptor 2 antibody), which, when administered in combination with taxane-based chemotherapy, significantly delayed PFS compared with taxane and placebo. ${ }^{22}$ Although these positive results may be drug specific, it is also possible that combination strategies (chemotherapy/VEGF-targeted therapy) are preferable to the single-agent strategy used in our study, although other such combinations have proven difficult to deliver. ${ }^{23}$

The second study of note relates to maintenance sunitinib administered after first-line chemotherapy, compared with placebo. This trial was stopped early, and the numbers were small. However, median PFS was 2.9 versus 2.7 months for sunitinib and placebo, respectively. Also, patients whose disease progressed on placebo crossed over to receive sunitinib $(n=16)$. These patients did not benefit from therapy, with only one response, suggesting limited activity for single-agent VEGF therapy in line with those seen in our study. ${ }^{24}$ The third study investigated paclitaxel with or without vandetanib, which is a broad-spectrum tyrosine kinase inhibitor (TKI) with VEGF receptor inhibition. This study showed no improvement in outcome associated with the addition of the targeted therapy. Together, these studies fail to form a consensus on the role of VEGF-targeted therapy in UC. ${ }^{25}$

It is possible that there are molecularly defined subgroups of patients who do benefit from VEGF-targeted therapy, or, conversely, who may be specifically sensitive to taxanes. As part of this trial, we have a comprehensive archival tissue and blood collection. 


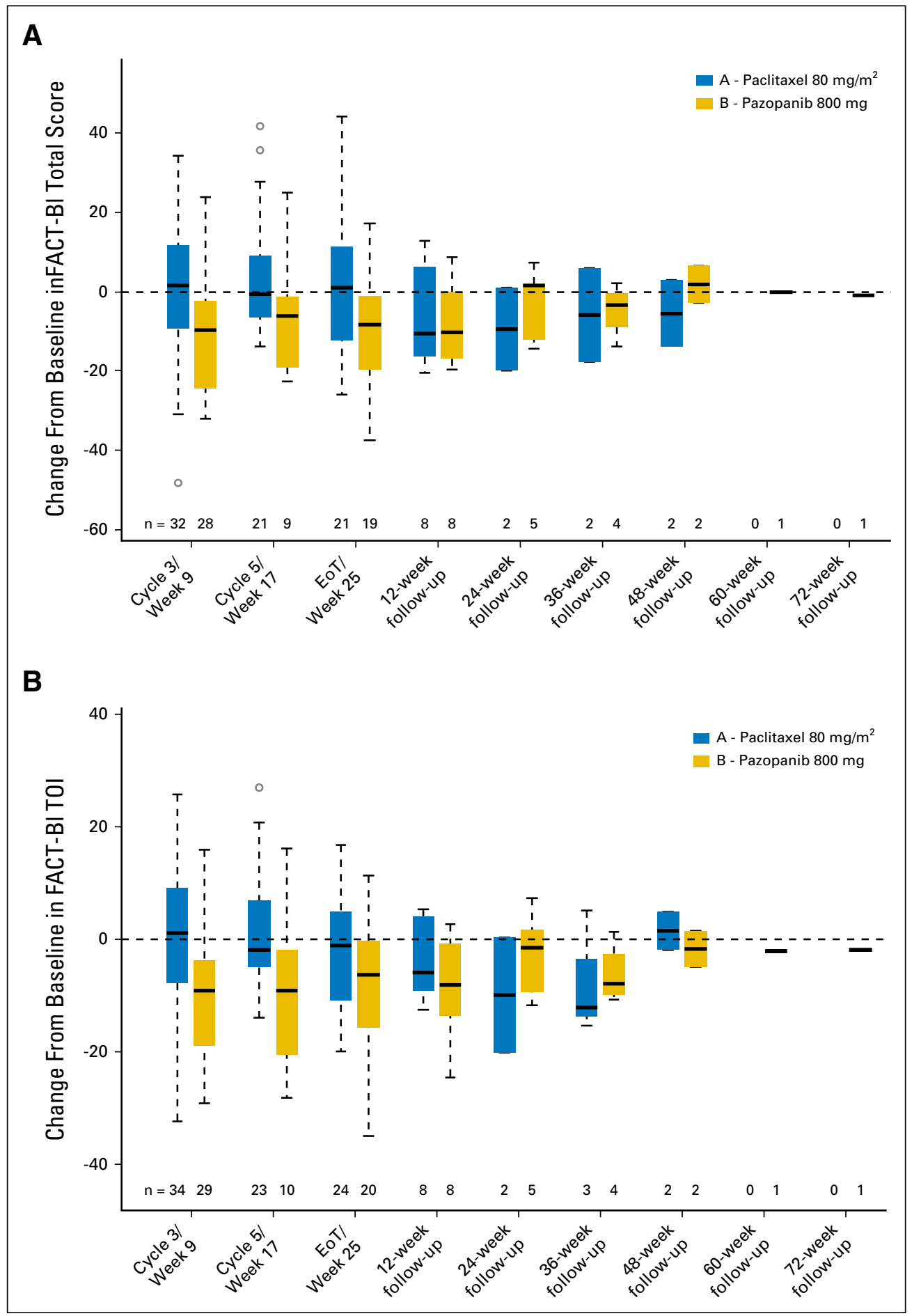

Fig 4. Quality of life analysis of change from baseline in (A) Functional Assessment of Cancer Therapy-Bladder (FACT-BI) total score, and (B) FACT-BI trial outcome index (TOI) by arm, assessed using area under the curve techniques. ${ }^{15}$ Number of data points is included. EoT, end of treatment.

Analyses are ongoing to establish putative predictive markers to differentiate those patients who may benefit from one or other of these approaches.

High levels of grade 3 or 4 toxicity occurred in both arms. This was numerically higher in the pazopanib arm. Specific toxicity was in line with expected toxicity associated with the respective drugs. Higher levels of discontinuation of study drug occurred with pazopanib (23\% $v 8 \%)$. Quality of life dropped from baseline in both arms, although more markedly with pazopanib. Overall, these results demonstrate the difficulty of giving systemic therapy in these patients and do not suggest pazopanib is easily tolerable in this setting.

No patients switched from pazopanib to paclitaxel when the study was stopped, despite the opportunity being offered to them. We speculate that this is a reflection of the perceived inactivity of chemotherapy in this setting. 
The study presented here conclusively showed that singleagent pazopanib should not be further pursued in unselected patients, resolving the previous controversy in this setting. Alternative approaches should not be excluded with this or other VEGF-targeted therapy. Also, a biomarker-based personalized approach should not be discounted, although VEGF-based biomarkers have been elusive.

\section{AUTHORS' DISCLOSURES OF POTENTIAL CONFLICTS OF INTEREST}

Disclosures provided by the authors are available with this article at jco.org.

\section{AUTHOR CONTRIBUTIONS}

Conception and design: Robert J. Jones, Prabir Chakraborti, Laura Alexander, Caroline Kelly, James Paul, Thomas Powles

Administrative support: Laura Alexander

Provision of study materials or patients: Robert J. Jones

Collection and assembly of data: Robert J. Jones, Syed A. Hussain, Robert A. Huddart, Satinder Jagdev, Amit Bahl, Andrew Stockdale, Santhanam Sundar, Judith Dixon-Hughes, Anna Morris, Thomas Powles

Data analysis and interpretation: Robert J. Jones, Andrew S. Protheroe, Alison Birtle, Santhanam Sundar, Simon J. Crabb, Caroline Kelly, Jon Stobo, James Paul, Thomas Powles

Manuscript writing: All authors

Final approval of manuscript: All authors

Accountable for all aspects of the work: All authors

\section{REFERENCES}

1. Alfred Witjes J, Lebret T, Compérat EM, et al: Updated 2016 EAU guidelines on muscle-invasive and metastatic bladder cancer. Eur Urol 71:462475, 2016

2. von der Maase $\mathrm{H}$, Hansen SW, Roberts JT, et al: Gemcitabine and cisplatin versus methotrexate, vinblastine, doxorubicin, and cisplatin in advanced or metastatic bladder cancer: Results of a large, randomized, multinational, multicenter, phase III study. J Clin Oncol 18:3068-3077, 2000

3. Sonpavde G, Sternberg CN, Rosenberg JE, et al: Second-line systemic therapy and emerging drugs for metastatic transitional-cell carcinoma of the urothelium. Lancet Oncol 11:861-870, 2010

4. Vaughn DJ, Broome CM, Hussain M, et al: Phase II trial of weekly paclitaxel in patients with previously treated advanced urothelial cancer. J Clin Oncol 20:937-940, 2002

5. Oing C, Rink M, Oechsle K, et al: Second line chemotherapy for advanced and metastatic urothelial carcinoma: Vinflunine and beyond-a comprehensive review of the current literature. J Urol 195:254-263, 2016

6. Bellmunt $\mathrm{J}$, Théodore $\mathrm{C}$, Demkov $\mathrm{T}$, et al: Phase III trial of vinflunine plus best supportive care compared with best supportive care alone after a platinum-containing regimen in patients with advanced transitional cell carcinoma of the urothelial tract. J Clin Oncol 27:4454-4461, 2009

7. Hahn NM, Stadler WM, Zon RT, et al: Phase II trial of cisplatin, gemcitabine, and bevacizumab as first-line therapy for metastatic urothelial carcinoma: Hoosier Oncology Group GU 04-75. J Clin Oncol 29: 1525-1530, 2011

8. Mazzola CR, Chin J: Targeting the VEGF pathway in metastatic bladder cancer. Expert Opin Investig Drugs 24:913-927, 2015
9. Motzer RJ, Hutson TE, Cella D, et al: Pazopanib versus sunitinib in metastatic renal-cell carcinoma. N Engl J Med 369:722-731, 2013

10. Necchi A, Mariani L, Zaffaroni N, et al: Pazopanib in advanced and platinum-resistant urothelial cancer: An open-label, single group, phase 2 trial. Lancet Oncol 13:810-816, 2012

11. Pili R, Qin R, Flynn PJ, et al: A phase II safety and efficacy study of the vascular endothelial growth factor receptor tyrosine kinase inhibitor pazopanib in patients with metastatic urothelial cancer. Clin Genitourin Cancer 11:477-483, 2013

12. Egan $P$, Venugopal $B$, Jones RJ: Chemotherapy for bladder cancer: A United Kingdom practice survey. J Clin Oncol 26:20s, 2008 (suppl; abstr 16078)

13. Freedman LS, White SJ: On the use of Pocock and Simon's method for balancing treatment numbers over prognostic factors in the controlled clinical trial. Biometrics 32:691-694, 1976

14. Rubinstein LV, Korn EL, Freidlin B, et al: Design issues of randomized phase II trials and a proposal for phase II screening trials. J Clin Oncol 23: 7199-7206, 2005

15. Hong $S$, Wang $Y$ : A three-outcome design for randomized comparative phase II clinical trials. Stat Med 26:3525-3534, 2007

16. Qian W, Parmar MK, Sambrook RJ, et al: Analysis of messy longitudinal data from a randomized clinical trial. Stat Med 19:2657-2674, 2000

17. Benjamini $Y$, Hochberg M: Controlling the false discovery rate: A practical and powerful approach to multiple testing. J R Stat Soc B 57:289-300, 1995

18. $\mathrm{Xu} L, W u X, \mathrm{Hu} C$, et al: A meta-analysis of combination therapy versus single-agent therapy in anthracycline- and taxane-pretreated metastatic breast cancer: Results from nine randomized phase III trials. Onco Targets Ther 9:4061-4074, 2016
19. Powles $T$, Eder JP, Fine GD, et al: MPDL3280A (anti-PD-L1) treatment leads to clinical activity in metastatic bladder cancer. Nature 515: 558-562, 2014

20. Rosenberg JE, Hoffman-Censits J, Powles T, et al: Atezolizumab in patients with locally advanced and metastatic urothelial carcinoma who have progressed following treatment with platinum-based chemotherapy: A single-arm, multicentre, phase 2 trial. Lancet 387:1909-1920, 2016

21. Bellmunt $J$, de Wit $R$, Vaughn $D J$, et al: KEYNOTE-045: Open-label, phase 3 study of pembrolizumab versus investigator's choice of paclitaxel, docetaxel, or vinflunine for previously treated advanced urothelial cancer. Presented at the Society for Immunotherapy of Cancer, National Harbor, MD, November 9-13, 2016 (abstr 470)

22. Petrylak DP, Tagawa ST, Kohli $M$, et al: Docetaxel as monotherapy or combined with ramucirumab or icrucumab in second-line treatment for locally advanced or metastatic urothelial carcinoma: An open-label, three-arm, randomized controlled phase II trial. J Clin Oncol 34:1500-1509, 2016

23. Geldart T, Chester J, Casbard A, et al: SUCCINCT: An open-label, single-arm, non-randomised, phase 2 trial of gemcitabine and cisplatin chemotherapy in combination with sunitinib as first-line treatment for patients with advanced urothelial carcinoma. Eur Urol 67:599-602, 2015

24. Grivas PD, Daignault $S$, Tagawa $S T$, et al: Double-blind, randomized, phase 2 trial of maintenance sunitinib versus placebo after response to chemotherapy in patients with advanced urothelial carcinoma. Cancer 120:692-701, 2014

25. Choueiri TK, Ross RW, Jacobus $S$, et al: Double-blind, randomized trial of docetaxel plus vandetanib versus docetaxel plus placebo in platinum-pretreated metastatic urothelial cancer. J Clin Oncol 30:507-512, 2012

\section{Affiliations}

Robert J. Jones, Judith Dixon-Hughes, Laura Alexander, Anna Morris, Caroline Kelly, Jon Stobo, and James Paul, University of Glasgow, Glasgow; Syed A. Hussain, University of Liverpool, Liverpool; Andrew S. Protheroe, Churchill Hospital, Oxford; Alison Birtle, Royal Preston Hospital, Preston; Prabir Chakraborti, Royal Derby Hospital, Derby; Robert A. Huddart, Institute of Cancer Research, Sutton; Satinder Jagdev, St James's University Hospital, Leeds; Amit Bahl, Bristol Haematology and Oncology Centre, Bristol; Andrew Stockdale, University Hospital, Coventry; Santhanam Sundar, Nottingham University Hospitals National Health Service Trust, Nottingham; Simon J. Crabb, University of Southampton, Southampton; and Thomas Powles, Queen Mary University of London, London, United Kingdom. 


\section{AUTHORS' DISCLOSURES OF POTENTIAL CONFLICTS OF INTEREST}

Randomized Phase II Study Investigating Pazopanib Versus Weekly Paclitaxel in Relapsed or Progressive Urothelial Cancer

The following represents disclosure information provided by authors of this manuscript. All relationships are considered compensated. Relationships are self-held unless noted. I = Immediate Family Member, Inst = My Institution. Relationships may not relate to the subject matter of this manuscript. For more information about ASCO's conflict of interest policy, please refer to www.asco.org/rwc or ascopubs.org/jco/site/ifc.

Robert J. Jones

Honoraria: Novartis, GlaxoSmithKline, Bristol-Myers Squibb

Consulting or Advisory Role: Roche, Merck Sharp \& Dohme, BristolMyers Squibb

Research Funding: Novartis, GlaxoSmithKline, Roche, Merck Sharp \& Dohme

\section{Syed A. Hussain}

Consulting or Advisory Role: Pierre Fabre, Roche, Merck, AstraZeneca, Bayer AG, Janssen Oncology

Research Funding: Boehringer Ingelheim

Travel, Accommodations, Expenses: Janssen-Cilag, Merck, Pierre Fabre, Janssen Oncology

Andrew S. Protheroe

Consulting or Advisory Role: Astellas Pharma, Bayer AG, Ipsen, Pfizer Travel, Accommodations, Expenses: Bristol-Myers Squibb

Alison Birtle

Consulting or Advisory Role: Roche, Janssen, Sanofi, Astellas Pharma, AstraZeneca

Prabir Chakraborti

Honoraria: Roche, Astellas Pharma, Janssen

Research Funding: Eisai

Robert A. Huddart

Leadership: Cancer Centre London

Honoraria: Roche

Consulting or Advisory Role: Roche (I), Merck Sharp \& Dohme (I)

Research Funding: Merck Sharp \& Dohme

Travel, Accommodations, Expenses: Merck Sharp \& Dohme

Satinder Jagdev

Honoraria: Novartis

Travel, Accommodations, Expenses: Astellas Pharma

Amit Bahl

Honoraria: Sanofi, Janssen, Novartis, Bayer AG

Research Funding: Ipsen (Inst), Sanofi (Inst)

Travel, Accommodations, Expenses: Janssen, Bayer AG, Astellas

\section{Andrew Stockdale}

No relationship to disclose

\section{Santhanam Sundar}

No relationship to disclose

\section{Simon J. Crabb}

Honoraria: Bayer AG, Astellas Pharma

Consulting or Advisory Role: Bayer AG, Sanofi, Astellas Pharma, Janssen, Dendreon, Pfizer

Research Funding: AstraZeneca, Astex Pharmaceuticals, Plexxikon

Travel, Accommodations, Expenses: Astellas Pharma, Sanofi, Novartis, Janssen, Bayer AG

Judith Dixon-Hughes

No relationship to disclose

Laura Alexander

No relationship to disclose

Anna Morris

No relationship to disclose

\section{Caroline Kelly}

No relationship to disclose

Jon Stobo

No relationship to disclose

James Paul

No relationship to disclose

Thomas Powles

Honoraria: Genentech

Consulting or Advisory Role: Genentech, Bristol-Myers Squibb, Merck, Novartis, AstraZeneca

Research Funding: AstraZeneca/MedImmune, Genentech 


\section{Acknowledgment}

We thank Cancer Research UK Clinical Trials Unit (CRUK CTU) Glasgow, all participating sites (G. McDonald, Aberdeen Royal Infirmary; D. Mazhar, Addenbrookes Hospital, Cambridge; A. Elliott, The Christie Hospital, Manchester; B. Sizer, Essex County Hospital; A. Clarke, Darent Valley Hospital; N. Sarward, Imperial Healthcare Trust; G. Faust, Leicester Royal Infirmary; S. Beesley, Maidstone Oncology Centre; J. Frew, The Freeman Hospital, Newcastle; I. Sayers, New Cross Hospital; S. Brock, Royal Bournemouth Infirmary; L. Evans, Weston Park Hospital, Sheffield; M. Varughese, Musgrove Park Hospital, Taunton; A. Lydon, Torbay District General Hospital; J. Barber, Velindre Cancer Centre, Cardiff), National Health Services Greater Glasgow and Clyde, University of Glasgow, Orchid Tissue Bank, and Data Monitoring Committee (DMC) members (Iain McNeish, John Kelly, Angela Casbard). 